\title{
GeV $\gamma$-ray Emission Detected by Fermi-LAT Probably Associated with the Thermal Composite Supernova Remnant Kesteven 41 in a Molecular Environment
}

\author{
Bing Liu ${ }^{1}$, Yang Chen ${ }^{1,2,5}$, Xiao Zhang ${ }^{1}$, Gao-Yuan Zhang ${ }^{1}$, Yi Xing ${ }^{3}$, \& Thomas G. \\ Pannuti ${ }^{4}$
}

\begin{abstract}
Hadronic emission from supernova remnant (SNR)-molecular cloud (MC) association systems has been widely regarded as a probe of the shock-accelerated cosmic-ray protons. Here, we report on the detection of a $\gamma$-ray emission source with a significance of $24 \sigma$ in $0.2-300 \mathrm{GeV}$, projected to lie to the northwest of the thermal composite SNR Kesteven 41, using 5.6 years of Fermi-Large Area Telescope (LAT) observation data. No significant long-term variability in the energy range $0.2-300 \mathrm{GeV}$ is detected around this source. The $3 \sigma$ error circle, 0.09 in radius, covers the $1720 \mathrm{MHz} \mathrm{OH}$ maser and is essentially consistent with the location of the $V_{\mathrm{LSR}} \sim-50 \mathrm{~km} \mathrm{~s}^{-1} \mathrm{MC}$ with which the SNR interacts. The source emission has an exponential cutoff power-law spectrum with a photon index of $1.9 \pm 0.1$ and a cutoff energy of $4.0 \pm 0.9 \mathrm{GeV}$, and the corresponding $0.2-300 \mathrm{GeV}$ luminosity is $\sim 1.3 \times 10^{36} \mathrm{erg} \mathrm{s}^{-1}$ at a distance of $12 \mathrm{kpc}$. There is no radio pulsar in the $3 \sigma$ circle responsible for the high $\gamma$-ray luminosity. While the inverse Compton scattering scenario would lead to a difficulty in the electron energy budget, the source emission can naturally be explained by the hadronic interaction between the relativistic protons accelerated by the shock of SNR Kesteven 41 and the adjacent northwestern MC. In this paper, we present a list of Galactic thermal composite SNRs detected at GeV $\gamma$-ray energies by Fermi-LAT is presented.
\end{abstract}

\footnotetext{
${ }^{1}$ Department of Astronomy, Nanjing University, 163 Xianlin Avenue, Nanjing 210023, China

${ }^{2}$ Key Laboratory of Modern Astronomy and Astrophysics, Nanjing University, Ministry of Education, Nanjing 210093, China

${ }^{3}$ Key Laboratory for Research in Galaxies and Cosmology, Shanghai Astronomical Observatory, Chinese Academy of Sciences, 80 Nandan Road, Shanghai 200030, China

${ }^{4}$ Space Science Center, Department of Earth and Space Sciences, Morehead State University, 235 Martindale Drive, Morehead, KY 40351, USA

${ }^{5}$ Corresponding author.
} 
Subject headings: acceleration of particles — gamma rays: ISM — ISM: supernova remnants — ISM: individual objects (Kes 41)

\section{INTRODUCTION}

The origin of the cosmic rays (CRs) remains one of the most debated issues in high energy astrophysics more than 100 years after they were detected for the first time. Supernova remnants (SNRs), whose strong shocks contain huge amounts of energy, are considered to be the most probable candidates among the Galactic CR acceleration sources (e.g., Ginzburg \& Syrovatskii 1969). A long standing argument concerning the putative SNR-CR link is that non-thermal radio emission from SNRs provides clear evidence for electron acceleration, whereas the CR spectrum observed on Earth is $99 \%$ protons and other nuclei. Therefore, the $\gamma$-ray emission from SNRs that is dominated by the decay of $\pi^{0}$ mesons produced via proton-proton collisions (i.e., the hadronic interaction) plays a key role in providing evidence for proton acceleration (Ackermann et al. 2013). However, it is often difficult to distinguish between the hadronic $\gamma$-ray emission and the electrons' inverse Compton or non-thermal bremstrahlung emission (i.e., the leptonic emission). There are generally two scenarios that describe how hadronic $\gamma$-rays are produced in SNRs. In one scenario, the $\pi^{0}$ decay emission is suggested to arise from shock-crushed dense clouds where the accelerated protons frozen in the clouds efficiently collide with target cloud gas (e.g., Blandford \& Cowie 1982; Uchivama et al. 2010; Tang \& Chevalier 2014). In the other scenario, the hadronic $\gamma$ rays are ascribed to interactions between the relativistic protons escaping from the SNR shock and adjacent molecular clouds (MCs; Aharonian \& Atoyan e.g., 1996; Gabici et al. e.g., 2009; Li \& Chen e.g., 2010; Ohira et al. e.g., 2011). In both scenarios, the SNRs interacting with MCs are crucial probes in the search for the signatures of proton acceleration. The hadronic $\gamma$-ray emission from SNR-MC systems is usually bright around GeV, and a series of GeV-bright SNRs interacting with MCs have recently been discovered with the Large Area Telescope (LAT) on board the Fermi Gamma-ray Space Telescope. These SNRs include W51C (Abdo et al. 2009), W44 (Abdo et al. 2010c), IC 443 (Abdo et al. 2010d), W28 (Abdo et al. 2010a), W41 (Castro et al. 2013), RCW 103 (Xing et al. 2014), etc. Additional $\mathrm{GeV}$ observations continue to enlarge the sample of hadronic interaction between SNRs and MCs, and here we present a GeV study of another SNR, namely, Kesteven 41(G337.8-0.1).

As a southern-sky SNR, Kes 41 is shown to be centrally brightened in X-rays within a distorted radio shell by a XMM-Newton observation (Combi et al. 2008), and therefore is classified as a thermal composite (or mixed-morphology) SNR (Jones et al. 1998; Rho \& Petre 1998). The X-ray emitting plasma of the SNR has been newly revealed to be rich in sulfur 
and argon; thus, Kes 41 joins the subclass of "enhanced-abundance" or "ejecta-dominated" thermal composites (Zhang et al. 2015). Kes 41 has also been found to be interacting with an adjacent $\mathrm{MC}$, as indicated by the $1720 \mathrm{MHz}$ hydroxyl radical $(\mathrm{OH})$ maser emission detected in the northern radio shell (Koralesky et al. 1998; Caswell 2004). Recently, we found that Kes 41 is associated with a giant MC at a systemic local standard of rest (LSR) velocity of $-50 \mathrm{~km} \mathrm{~s}^{-1}$ and is confined in a cavity delineated by a northern molecular shell, a western concave MC, and a southeastern H I cloud (Zhang et al. 2015). The forward shock is suggested to have left the adiabatic stage since the SNR shock encountered the cavity wall, while the inner thermal X-rays are ascribed to heating by the reflection shock from the cavity wall. The birth of Kes 41 inside the molecular cavity provides a mass estimate of $\gtrsim 18 M_{\odot}$ for the stellar progenitor. It is logical and meaningful to search for the hadronic emission due to the interaction of the SNR with the dense environmental gas.

In this paper, we report the results from a spatial and spectral analysis of the FermiLAT observation data of the Kes 41 region. We describe the Fermi observation data in Section 2, and present the data analysis and results in Section 3. The possible physical relation of the detected $\gamma$-ray emission with the SNR is discussed in Section 4 .

\section{OBSERVATIONS AND DATA REDUCTION}

The LAT on board Fermi, launched on 2008 June 11, is a $\gamma$-ray imaging instrument that covers a very wide range of energy from $20 \mathrm{MeV}$ and up to $300 \mathrm{GeV}$. It reconstructs the direction of incident $\gamma$-rays by tracking the electrons and positrons resulting from pair conversion of the $\gamma$-rays in the solid state silicon trackers, and measures the energy of the subsequent electromagnetic showers that develop in the cesium iodide calorimeters (Atwood et al. 2009). The point-spread function (PSF) varies largely with photon energy and improves at high energies (the $68 \%$ containment radius at $>2 \mathrm{GeV}$ is smaller than 0.5 Atwood et al. 2009).

We use the reconstructed Pass 7 reprocessed version of 5.6 years of accumulated FermiLAT datal that has been selected from 2008 August 04 15:43:37 (UTC) to 2014 April 01 02:29:28 (UTC). We analyze the data with the standard software, ScienceTools version v9r32p52 released on 2013 October 24, with the instrument response functions (IRFs) P7REP_SOURCE_V15. Standard selection criteria are applied to the data selection process as described below. The Source (evclass=2) events are selected and the maximum zenith

\footnotetext{
${ }^{1}$ http://fermi.gsfc.nasa.gov/ssc/data

${ }^{2}$ See http://fermi.gsfc.nasa.gov/ssc
} 
angle cut is $100^{\circ}$ to reduce the residual $\gamma$-rays from CR interactions in the upper atmosphere. We used the standard criteria for selecting time intervals for analysis: (DATA_QUAL==1) $\& \&($ LAT_CONFIG $==1) \& \&$ ABS(ROCK_ANGLE) $<52$. The analysis is restricted to the energy range above $200 \mathrm{MeV}$ due to uncertainties in the effective area and broad PSF at low energies and below $300 \mathrm{GeV}$ due to limited statistics.

\section{ANALYSIS AND RESULTS}

In our analysis, we select the LAT events inside a $14^{\circ} \times 14^{\circ}$ region of interest (ROI, in equatorial coordinate system) centered at the position of the Kes 41 (R.A. (J2000) = $16^{\mathrm{h}} 39^{\mathrm{m}} 00^{\mathrm{s}}$ and decl. $\left.(\mathrm{J} 2000)=-46^{\circ} 58^{\prime} 59^{\prime \prime}\right)$ with a bin size of $0^{\circ} .04 \times 0^{\circ} .04$. We perform our analysis following the standard binned likelihood analysis procedure. The second Fermi-LAT Catalog (2FGL) sources (Nolan et al. 2012) within radius $15^{\circ}$ around Kes 41 are included in the source model, which was generated by the user-contributed software make2FGLxml py $3^{3}$. The Galactic and extragalactic diffuse background components (as specified in the files gll_iem_v05.fits and iso_source05.txt, respectively) are used. In the likelihood fittings, the spectral parameters of the sources located beyond $10^{\circ}$ of the ROI center are fixed to the values reported in 2FGL, and the spectral parameters of all the sources located within $10^{\circ}$ of the center of ROI, together with the normalizations of the two diffuse backgrounds, are allowed to vary. The fittings are performed with the optimizer NEWMINUIT until convergence is achieved.

\subsection{Source Detection}

First, a binned likelihood analysis is applied in the energy range $2-300 \mathrm{GeV}$. In the source model, the source 2FGL J1638.0-4703c, which is very close to Kes 41, has been removed due to the uncertainty of its spatial and spectral information caused by the imperfectly modeled diffuse emission, and thus needs to be treated with great care4 (Nolan et al. 2012). A newly

discovered $\gamma$-ray source (HESS J1641-463, Lemoine-Goumard et al. 2014) has been added assuming a power-law spectrum. Then, the test statistic $\left(\mathrm{TS}\right.$, defined as $2\left(\log \mathcal{L}-\log \mathcal{L}_{0}\right)$, here $\mathcal{L}_{0}$ is the likelihood of null hypothesis and $\mathcal{L}$ is the likelihood with the source included) map for a $1^{\circ} \times 1^{\circ}$ region centered at Kes 41 is made after subtracting this baseline model (see

\footnotetext{
${ }^{3}$ http://fermi.gsfc.nasa.gov/ssc/data/analysis/user/

${ }^{4}$ http://fermi.gsfc.nasa.gov/ssc/data/access/lat/2yr_catalog/
} 
Fig. 1). As can be seen in Figure 1, there is excess $\gamma$-ray emission in the region of Kes 41. The position of the peak of the TS value is on the northwest of the SNR, but does not agree with the position of 2FGL J1638.0-4703c. It is generally consistent with the location of the dense $\mathrm{MC}$ at $V_{\mathrm{LSR}} \sim-50 \mathrm{~km} \mathrm{~s}^{-1}$ which is found to be associated with the SNR (Zhang et al. 2015). We also perform an analysis in the low energy range $0.2-2 \mathrm{GeV}$, and some residual $\gamma$-ray emission is detected at the same position. Therefore, we add a point source with a power-law spectrum at the position where the TS value is highest in our source model to approximate the excess emission. After that, we conduct a binned likelihood analysis in the broad energy range $0.2-300 \mathrm{GeV}$ and, utilizing gtfindsrc (a tool in the LAT software package ScienceTools), we find the best-fit position of the excess $\gamma$-ray emission at (R.A. (J2000) $=$ $16^{h} 38^{m} 36^{s} .00$, decl. (J2000) $\left.=-46^{\circ} 55^{\prime} 06^{\prime \prime} .96\right)$ with $1 \sigma$ nominal uncertainty of 0.03 and $3 \sigma$ nominal uncertainty of 0.09 .

By comparison, we detect this source as a point-like source with a power-law spectrum in 0.2-300 GeV with a significance of $24 \sigma$ at the best-fit position and increases the significance by $1 \sigma$ over the position of 2 FGL J1638.0-4703c. The data we use are collected from 5.6 years of Fermi-LAT observations while the tentative source 2FGL J1638.0-4703c was suggested based on the first two years of observations. Both the statistical result and increased exposure time hence suggest that the $\gamma$-ray emission excess at the best-fit position adjacent to Kes 41 is more significant than 2FGL J1638.0-4703c. Thus, we replace 2FGL J1638.0-4703c with this new source at the best-fit position (hereafter source A) in the following analysis.

In an attempt to explore the origin of the $\gamma$-ray emission of source $A$, we searched in the SIMBAD Astronomical Database (Wenger et al. 2000) within a $3 \sigma$ error circle of the source (see Fig. 1). In addition to SNR Kes 41, only nine dark clouds, a young stellar object candidate, and an infrared source are known to exist in the region. Therefore, the origin of this $\gamma$-ray emission is most likely related to the SNR.

\subsection{Timing Analysis}

We next search for long-term variability in the one month binned light curve of source A in the energy range $0.2-300 \mathrm{GeV}$, which is obtained from likelihood analysis (Nolan et al. 2012) in each time bin. As can be seen in the light curve (Figure 21), all of the flux points remain within $3 \sigma$ uncertainties of the average flux. Fitting the flux points with TS value $>4$ to a constant flux model (shown as a red line in Figure 2) yields a $\chi^{2} \sim 32.3$ with 48 degrees of freedom (dof). Moreover, we calculate the Variability_Index, $\mathrm{TS}_{\mathrm{var}}$, of source A (with all 69 time bins) in the $0.2-300 \mathrm{GeV}$ energy range according to the method introduced in $\S 3.6$

of Nolan et al. (2012). If the flux is constant, then $\mathrm{TS}_{\mathrm{var}}$ is distributed as $\chi^{2}$ with 68 dof, 
and variability would be considered probable when $\mathrm{TS}_{\text {var }}$ could exceed the threshold of 98.0 corresponding to $99 \%$ confidence. The computed $\mathrm{TS}_{\text {var }}$ of source A is 65.2 , corresponding to a confidence level $<50 \%$ for a variable source. These results suggest that there is no signifiant long-term variability observed in the region of Source A in the $0.2-300 \mathrm{GeV}$ energy range. On the other hand, we construct $1000 \mathrm{~s}$ binned light curves of source A in the same energy range which are obtained through Fermi-LAT aperture photometry analyses 5 using LAT photons within different aperture radius from 0.2 to 0.5 . We analyze these light curves for periodic signals, but no significant periodicity is detected. However, this method is statistically limited and the periodicity is hard to detect due to the massive diffuse background photons in a low galactic latitude.

There is a close positional correspondence between source A, suggested here as a steady source, and 3FGL J1638.6-4654, which is indicated as a variable source in the third FermiLAT Catalog (3FGL) (Acero et al. 2015). 3FGL J1638.6-4654 is detected in 0.1-300 GeV with a significance of $13 \sigma$ and a $0.1-300 \mathrm{GeV}$ energy flux $\sim 7.3 \times 10^{-11} \mathrm{erg} \mathrm{cm}^{-2} \mathrm{~s}^{-1}$; and source A has a higher significance $(24 \sigma)$ with an energy flux of $\sim 7.5 \times 10^{-11} \mathrm{erg} \mathrm{cm}^{-2} \mathrm{~s}^{-1}$ in the $0.2-300 \mathrm{GeV}$ energy range (see Section 4.1; the flux will be somewhat higher in $0.1-$ $300 \mathrm{GeV}$ ). The use of different spectral models and different energy ranges in the two timing analyses may contribute to the discrepancy in the variability between source A and 3FGL J1638.6-4654. The spectrum of source A is fit to a power-law model and the spectrum of 3FGL J1638.6-4654 is fit to a log-parabola model. Moreover, our timing analysis of source A uses photons in the energy range $0.2-300 \mathrm{GeV}$ while 3FGL J1638.6-4654 is analyzed in the energy range $0.1-300 \mathrm{GeV}$. Our timing analysis would not be sensitive to flux variations (if any) below $0.2 \mathrm{GeV}$.

\subsection{Spatial Distribution Analysis}

We analyze the spatial distribution of source A, which is very likely to be associated with Kes 41, to examine whether it is a point-like or extended source. We apply both point-source and uniform-disk models with power-law spectra at the best-fit position to fit the emission in the energe range $2-300 \mathrm{GeV}$. In the point-source case, we set the spectral normalizations of the sources within $10^{\circ}$ of Kes 41 as free parameters, and fix all the other parameters at the 2FGL values. A TS value of 207 is obtained. In the disk case, the observed radius range for the uniform disks is $0.1-0.5$ with a step of 0.1 . We fix all of the spectral parameters of the sources at the values obtained above, but allow the spectral normalization parameters of the

\footnotetext{
${ }^{5}$ http://fermi.gsfc.nasa.gov/ssc/data/analysis/scitools/aperture_photometry.html
} 
disk models to be free parameters. The $\mathrm{TS}_{\text {ext }}$ value (calculated from $2 \log \left(\mathcal{L}_{\text {disk }} / \mathcal{L}_{\text {point }}\right)$ ) for each radius is smaller than zero, while the extended source detection threshold is $\mathrm{TS}_{\text {ext }}=16$ (Lande et al. 2012), which implies that no significant extended emission is detected. As a result, the $\mathrm{GeV} \gamma$-ray emission from source $\mathrm{A}$ seems to be point-like.

\subsection{Spectral Analysis}

The $\gamma$-ray spectrum of source $A$ is extracted via the maximum likelihood analysis of the LAT data in 6 divided energy bands from $0.2-300 \mathrm{GeV}$ (see Table 1). The spectral normalization parameters of the sources within $5^{\circ}$ of Kes 41 are allowed to vary, but all of the other source parameters are fixed. In addition to the statistical uncertainties associated with the likelihood fits to the data, the uncertainty of the Galactic diffuse background intensity is considered. We vary the normalization of the Galactic background by $\pm 6 \%$ from the best-fit values at each energy bin and estimate the flux from the object of interest using these new artificially frozen values of the background, following the treatment in Abdo et al. (2009). The possible systematic errors are estimated to be $46 \%(0.2-0.5 \mathrm{GeV}), 40 \%(0.5-1.0 \mathrm{GeV})$, $20 \%(1.0-3.0 \mathrm{GeV})$, and $<15 \%(>3 \mathrm{GeV})$. We keep only spectral flux points with TS higher than 4 (which corresponds to the detection significance of $2 \sigma$ ) and derive $95 \%$ flux upper limits in the other energy bins. The obtained spectral data for source A are provided in Table 1.

We fit the $0.2-300 \mathrm{GeV}$ spectral data of source A with a power-law model. The obtained spectral shape is relatively flat with a photon index of $\Gamma=2.38 \pm 0.03$. The flux is $(9.2 \pm$ 1.0) $\times 10^{-11} \mathrm{erg} \mathrm{cm}^{-2} \mathrm{~s}^{-1}$, corresponding to a luminosity of $\sim 1.6 \times 10^{36} \mathrm{~d}_{12}^{2} \mathrm{erg} \mathrm{s}^{-1}$, where $d_{12}=d / 12 \mathrm{kpc}$ is the distance to the MC associated with SNR Kes 41 in units of the referecnce value estimated from the maser observation (Koralesky et al. 1998). Also see Section 4.1 for an estimate of the flux and luminosity with an exponential cutoff.

\section{DISCUSSION ON THE NATURE OF SOURCE A}

Based on our analysis of 5.6 years of Fermi-LAT data for the environment surrounding Kes 41 , we have found a $\gamma$-ray source detected at a significance of $\sim 24 \sigma$ that appears to be coincident with the northwest rim of Kes 41.

The relation between source A and Kes 41 is crucial for determining the origin of the $\gamma$-ray emission. In this section, we will discuss the possiblity of the $\gamma$-ray emission arising from a pulsar and an SNR-MC hadronic interaction, respectively. 


\subsection{A Pulsar?}

Galactic pulsars are important $\gamma$-ray source candidates, and there have been numerous pulsars detected by Fermi-LAT in recent years (Abdo et al. 2010e). Although the $3 \sigma$ error circle here does not include any known pulsars, the possibility of correspondence to a pulsar associated with Kes 41 still cannot be ignored. Theoretically, there may be a descendent stellar compact remnant after the core-collapse supernova (SN) explosion of the $\gtrsim 18 M_{\odot}$ progenitor of the remnant (Zhang et al. 2015). Such a compact stellar remnant has not been conclusively associated with Kes 41 in the literature.

We fit the spectrum of source A with a power-law model with an exponential cutoff, $d N_{\mathrm{ph}} / d E_{\mathrm{ph}}=K E_{\mathrm{ph}}^{-\Gamma} \exp \left(-E_{\mathrm{ph}} / E_{\mathrm{ph}, \mathrm{cut}}\right)$, typical for a pulsar (Abdo et al. 2010e). The model fit yields $E_{\mathrm{ph} \text {,cut }}=4.0 \pm 0.9 \mathrm{GeV}$ and the spectral index of $\Gamma=1.9 \pm 0.1$. In this model, the flux in energy range $0.2-300 \mathrm{GeV}$ is $(7.5 \pm 0.9) \times 10^{-11} \mathrm{erg} \mathrm{cm}^{-2} \mathrm{~s}^{-1}$, and the corresponding luminosity is $(1.3 \pm 0.2) \times 10^{36} d_{12}^{2} \mathrm{erg} \mathrm{s}^{-1}$. The significance of the exponential cutoff power law (approximately described by $\sqrt{T S_{\text {cutoff }}} \sigma=\sqrt{T S_{P L+\text { cutoff }}-T S_{P L}} \sigma$ ) is $\sim 6 \sigma$. The spectral shape of source A is similar to those of the detected $\gamma$-ray pulsars (Abdo et al. 2013), which usually show flat spectra below $1 \mathrm{GeV}$ and exponential cutoffs in the energy range $\sim 0.4^{-}$ $6 \mathrm{GeV}$. If this source is a "kicked" pulsar moving from the SNR center, then the best-fit position, $0 .^{\circ} 1$ away, would imply a projected traverse velocity of $180-4900 d_{12} \mathrm{~km} \mathrm{~s}^{-1}$ if the remnant's age estimate 4-110 kyr (Zhang et al. 2015) is adopted. (The closer the position within the $3 \sigma$ circle is to the SNR center, the lower the velocity would be.) The upper limit of the velocity seems very high, but there is also acuumulating evidence for high pulsar velocities, even exceeding $4 \times 10^{3} \mathrm{~km} \mathrm{~s}^{-1}$ (e.g., PSR B2011+38 and PSR B1718-35, Zou et al. 2005). On the other hand, if it is an associated pulsar at $\sim 12 \mathrm{kpc}$, then its $\gamma$-ray luminosity of the order of $10^{36} \mathrm{erg} \mathrm{s}^{-1}$ (Section 3.4) would be among the highest among the (radio loud) $\gamma$-ray pulsars (Abdo et al. 2013), which seems difficult to accept in view of the no detection of any radio pulsar here.

\subsection{Emission from Particles Accelerated by Kes 41?}

The $3 \sigma$ error circle is on the northwestern boundary of SNR Kes 41 and essentially consistent with the shock-MC interaction region. Actually, it covers not only the $1720 \mathrm{MHz} \mathrm{OH}$ maser but also the northwestern molecular gas at a systemic velocity of $V_{\mathrm{LSR}} \sim-50 \mathrm{~km} \mathrm{~s}^{-1}$ that surrounds the remnant (Zhang et al. 2015; see Fig. 3). It is very possible that the $\gamma$-ray emission arises from the relativistic particles accelerated by the SNR shock waves. We need to confront the leptonic and hardronic mechanisms with the obtained $\gamma$-ray data. 


\subsubsection{Leptonic Scenario}

First, we consider the scenario in which the $\gamma$-ray emission comes from the inverse Compton scattering off the relativistic electrons accelerated by the SNR shock. The emissivity of the bremsstrahlung process is compatible with that of the $p$ - $p$ process if the number ratio of electrons to protons at a given energy, $K_{e p}$, is of order $\sim 0.1$ (Gaisser et al. 1998).

Nevertheless, the values of $K_{e p}$ observed at Earth (Yuan et al. 2012) and predicted by the diffusive shock acceleration theory (Bell 1978) are both of the order of $\sim 0.01$. Therefore, the bremsstrahlung gamma-ray is usually insignificant.

We fit a power-law electron spectrum with a cutoff, $d N_{\mathrm{e}} / d E_{\mathrm{e}} \propto E_{\mathrm{e}}^{-\alpha_{e}} \exp \left(-E_{\mathrm{e}} / E_{\mathrm{e}, \text { cut }}\right)$, to the spectral data and only consider the cosmic microwave background as the seed photons (referred to as Case A). As can be seen in Fig. 4 (blue dotted line), the fitting effect is less satisfactory. We obtain $\alpha_{e} \approx 2.0$ and $E_{\mathrm{e}, \text { cut }} \approx 400 \mathrm{GeV}$ (also see Table 2). The normalization is given by the total energy deposited in electrons with energy above $1 \mathrm{GeV}$, $W_{\mathrm{e}}(>1 \mathrm{GeV}) \sim 1.3 \times 10^{51} \mathrm{erg}$. This electron energy budget is unreasonably high as the order of the canonical SN explosion energy.

\subsubsection{Hadronic Scenario}

Next, we consider the scenario in which the $\gamma$-ray emission is produced by the collision of the shock accelerated protons with dense molecular gas. For the case (referred to as Case $B$ ) in which the protons collide with the dense target molecular gas (with average number density $\left.n_{\mathrm{t}}\right)$, we assume for the protons a broken power-law distribution, $d N_{\mathrm{p}} / d E_{\mathrm{p}} \propto E_{\mathrm{p}}^{-\alpha_{\mathrm{p}}}(1+$

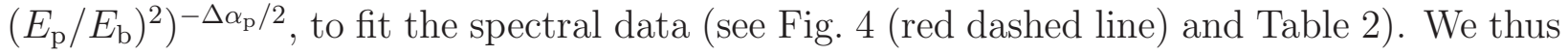
obtain $\alpha_{\mathrm{p}}=2.0, \Delta \alpha_{\mathrm{p}}=1.2$, and a break energy of $E_{\mathrm{b}}=18 \mathrm{GeV}$. The total energy deposited in the protons with energy above $1 \mathrm{GeV}$ is $W_{\mathrm{p}}(>1 \mathrm{GeV}) \sim 0.7 \times 10^{50} E_{51}\left(n_{\mathrm{t}} / 100 \mathrm{~cm}^{-3}\right)^{-1}$ erg, where $E_{51}=E_{\mathrm{SN}} / 10^{51} \mathrm{erg}$ is the dimensionless SN explosion energy. SNR Kes 41 has been found to be surrounded by molecular gas of density with $n_{\mathrm{H}_{2}} \sim 140-500 \mathrm{~cm}^{-3}$ in the northwest and HI gas of density with $n(\mathrm{HI}) \sim 40 \mathrm{~cm}^{-3}$ in the southeast (also see Fig. 3). If the mean target density $n_{\mathrm{t}}$ is approximately of the order of $100 \mathrm{~cm}^{-3}$, then $W_{\mathrm{p}}(>1 \mathrm{GeV}) \sim 1 \times 10^{50} E_{51} \mathrm{erg}$, namely, the fraction, $\eta$, of the SN explosion energy converted to protons is of the typical order of 0.1 . While in this scenario the hadronic $\gamma$-rays are emitted at the SNR shock, it is noteworthy that the centroid of the $3 \sigma$ circle of source A appears to be outside the northwestern boundary of the SNR.

The hadronic emission can alternatively be considered as originating from the adjacent MCs that are "illuminated" by the diffusive relativistic protons escaping from the SNR shock 
front. In the finite volume of a nearby cloud, the protons' energy distribution can be obtained by calculating the diffusive escaping protons accumulatively throughout the history of the SNR expansion (Case C). For such a calculation, in the following, we refer to Li \& Chen (2012) and the references therein for details of the model.

In the model calculation, we assume a converted CR proton energy fraction of $\eta=0.1$ and an SN explosion energy of $E_{\mathrm{SN}}=10^{51} \mathrm{erg}$. The SNR radius in the southeast-northwest orientation is adopted as $R_{s} \approx 11 \mathrm{pc}$. The $\gamma$-rays are assumed to arise from an MC, of thickness $\Delta R_{c}$, which is in contact with the shock surface; therefore, the MC center is at $R_{c}=R_{s}+\Delta R_{c} / 2$ away from the SNR center. According to Zhang et al. (2015), the SNR evolves in a cavity and may have been drastically decelerated and entered the radiative phase as soon as the blast wave encountered the cavity wall, after a Sedov evolution lifetime (Sedov 1959) of $t_{\mathrm{enc}}=4 \times 10^{3}\left(n_{\mathrm{H}} / 0.3 \mathrm{~cm}^{-3}\right)^{1 / 2} E_{51}^{-1 / 2}\left(R_{s} / 11 \mathrm{pc}\right)^{5 / 2}$ years. We assume that the particle acceleration process is not significant after this time. Therefore, the average distribution of the cumulative escaping protons in the volume of the MC at the remnant age $t_{\text {age }}$ is rewritten as

$$
\begin{aligned}
F_{\text {ave }}\left(E_{\mathrm{p}}, t_{\text {age }}\right)= & \int_{R_{c}-\Delta R_{c} / 2}^{R_{c}+\Delta R_{c} / 2} r^{2} d r \int_{0}^{t_{\mathrm{enc}}} \int_{0}^{2 \pi} \int_{0}^{\pi} f\left(E_{\mathrm{p}}, R_{\mathrm{bet}}\left(R_{\mathrm{c}}, t_{i}, \theta, \phi\right), t_{\mathrm{dif}}\right) R_{\mathrm{s}}^{2}\left(t_{i}\right) \sin \theta d \theta d \phi d t_{i} \\
& / \int_{R_{c}-\Delta R_{c} / 2}^{R_{c}+\Delta R_{c} / 2} r^{2} d r
\end{aligned}
$$

where $t_{i}$ is the time at which a proton escapes from the SNR shock, $t_{\text {dif }}=t_{\text {age }}-t_{i}$ is the diffusion time after escape, $R_{\text {bet }}$ is the distance between the escape point on the shock surface and a given point in the cloud (with position angles $(\theta, \phi))$, and $f\left(E_{\mathrm{p}}, R_{\text {bet }}\left(R_{\mathrm{c}}, t_{i}, \theta, \phi\right), t_{\text {dif }}\right)$ is the distribution function at a given point of the protons that escape from the unit area at an arbitrary escape point. Considering the remnant's age range $\sim 4-100 \mathrm{kyr}$ estimated from the ionization timescale of the X-ray emitting gas (Zhang et al. 2015), we calculate the model with three age numbers, 4, 10, and $100 \mathrm{kyr}$. This model can fit the spectral points as well, as exemplified by the solid line for $t_{\text {age }}=10 \mathrm{kyr}$ in Figure 4 . The model parameters are listed in Table 2. The photon index $\alpha_{\mathrm{p}}=2.4$, the energy-dependent index of the diffusion coefficient $\delta=0.7$, and the correction factor of slow diffusion around the SNR $\chi \sim 0.01-0.1$ are in normal ranges. The $\Delta R_{c}$ value $\sim 5-13 \mathrm{pc}(\sim 0.02-0.06)$ implies that the MC involed in the $p$ - $p$ hadronic interaction is essentially within the $3 \sigma$ circle of source A. Note that such a source size is much smaller than the PSF size 0.5 of the Fermi-LAT at energies above $2 \mathrm{GeV}$, consistent with the above judgement of a point-like source. The "illuminated" MC mass $M_{\mathrm{cl}}$, around $10^{5} M_{\odot}$, seems reasonable as compared with the mass of the molecular gas "reservoir" in the northwest, which is no less than $\sim$ a few times $10^{5} M_{\odot}$, which is estimated from a limited field of view of the CO observation (Zhang et al. 2015). 
However, the cavity wall may send a reflected shock backward when the blast wave collides with it (Zhang et al. 2015). If the reflected shock can still effectively accelerate particles after the forward shock becomes radiative, then the situation would be more complicated than the above cases. For simplicity, we approximate this case as a continuous proton injection from the SNR center (Aharonian \& Atoyan 1996) (Case D). In this case, the MC is regarded as a point at $R_{c}$ from the SNR center and the same energy conversion fraction $\eta=0.1$ is adopted. We follow the algorithm described in Aharonian \& Atovan (1996) and fit the spectral data, as exemplified by the green dashed line for $t_{\text {age }}=10 \mathrm{kyr}$ and $R_{c}=20 \mathrm{pc}$ in Figure 4. These model results are generally similar to those of Case $C$, with a slightly harder model spectrum at $\gtrsim 100 \mathrm{GeV}$. For the three sets of parameters with $t_{\text {age }}=10$ and $100 \mathrm{kyr}$, we again have $\alpha_{\mathrm{p}}=2.4$ and $\delta=0.7$. The $\chi$ values are $\sim 0.05-0.5$ in a normal range. A higher mass of the "illuminated" part of $\mathrm{MC}$ than Case $C$ is required, but is still consistent with the MC mass estimate from the CO observation in the order of magnitude.

The hadronic scenarios, both the interaction at the shock (Case B) and the illumination by escaping protons (Case $C / D$ ), can generally explain the $\gamma$-ray properties of source A. The escape cases have harder model spectra at $\gtrsim 10 \mathrm{GeV}$ than the interaction-at-the-shock case. Further TeV observations will likely be of help to distinguish the two scenarios.

\section{COMPARISON WITH OTHER GeV-DETECTED SNRs IN MC ENVIRONMENTS}

We now present a brief discussion of Kes 41 within the context of other Galactic SNRs that have been detected at $\gamma$-ray energies. An intriguing trend has emerged in these studies where the Galactic SNRs that are known to be interacting with dense clouds and that are detected at (very) high energies also appear to exhibit contrasting morphologies in the X-ray and the radio. Specifically, these sources exhibit the shell-like radio morphologies that are characteristic of SNRs coupled with a center-filled X-ray morphology that is thermal in origin, and therefore belong to the class of thermal composite or mixed-morphology SNRs (also see Section 1). Actually, about half of the 36-37 known thermal composites have been found to be interacting with adjacent MCs (see Table 4 in Zhang et al. 2015). While the origin of these contrasting morphologies remains uncertain, it appears that the interaction between the SNRs and the dense clouds plays a crucial role. Proposed origins for these morphologies include the evaporation of shock-engulfed cloudlets, thermal conduction within the interior hot gas, and heating by the shock reflected from the wind-cavity wall; the reader is referred to Chen et al. (2008) and references therein for a detailed review of these proposed mechanisms.

We tabulate the thermal composite SNRs that have been detected at $\gamma$-ray energies by 
Fermi-LAT in Table 3, So far, there are 13 SNRs (including Kes 41) of this class that have associated $\mathrm{GeV} \gamma$-ray emission, and an additional six of them possibly have associated $\mathrm{GeV}$ $\gamma$-ray emission, as listed in Table 3. We can see that most of the GeV-detected thermal composites are in physical interaction with MCs.

In Table 3, we collect the photon indices in the GeV band and adopt the luminosities in, or convert them to, the $1-100 \mathrm{GeV}$ energy range for ease of comparison. The $\sim \mathrm{GeV}$ spectra of these SNRs are soft, with power-law photon indices of $\Gamma \geq 2.0$, in distinct contrast with the hard spectra $(\Gamma \sim 1.4-1.8$ ) of the supposed leptonic process dominated $\gamma$-ray SNRs, e.g., RX J0852.0-4622 (Tanaka et al. 2011) and RCW 86(Yuan et al. 2014). Except for HB 21 and Kes 27, the 1-100 GeV luminosities of the 13 identified GeV $\gamma$-ray sources are on the order of a few times $10^{35} \mathrm{erg} \mathrm{s}^{-1}$, which are significantly higher than those of the leptonic process dominated SNRs (e.g., $<10^{34} \mathrm{erg} \mathrm{s}^{-1}$ for RX J0852.0-4622, Tanaka et al. 2011; and RCW 86, Yuan et al. 2014). For exceptional cases of HB 21 and Kes 27, the low luminosities may be due to proton collisions with only a very small amount of dense clouds (e.g., Pivato et al. 2013; Xing et al. 2015). We note that where detailed modeling has been applied to the $\gamma$-ray spectra of these sources, hadronic models have generally proved to give better fits to the data than leptonic models (except for the uncertain cases of Kes 17 and HB 9).

These past $\gamma$-ray observations of thermal composite SNRs-including the observation of Kes 41 that is presented in this paper - have thus produced insights into how SNRs interact with MC and how SNRs accelerate CR particles. Additional $\gamma$-ray observations of thermal composites are necessary and timely to explore the relation between emission at these high energies and the origin of the contrasting morphologies that characterize SNRs of this type.

\section{Summary}

We perform an analysis of the $\gamma$-ray emission in a $14^{\circ} \times 14^{\circ}$ region centered on the thermal composite SNR Kes 41, using 5.6 years of Fermi-LAT observation data. We find a point-like source to the northwest of the SNR with a significance of $24 \sigma$ in $0.2-300 \mathrm{GeV}$. Neither significant long-term variability nor periodicity is detected from the timing analysis of source $\mathrm{A}$ in the same energy range. The $3 \sigma$ error circle, 0.09 in radius, covers the $1720 \mathrm{MHz}$ $\mathrm{OH}$ maser and is essentially consistent with the location of the $V_{\mathrm{LSR}} \sim-50 \mathrm{~km} \mathrm{~s}^{-1} \mathrm{MC}$ with which the SNR interacts. The source emission can be described by a power-law spectrum with an exponential cutoff with a photon index of $1.9 \pm 0.1$ and a cutoff energy of $4.0 \pm 0.9 \mathrm{GeV}$. The corresponding $0.2-300 \mathrm{GeV}$ flux is $(7.5 \pm 0.9) \times 10^{-11} \mathrm{erg} \mathrm{cm}^{-2} \mathrm{~s}^{-1}$, and the luminosity is $\sim 1.3 \times 10^{36} \mathrm{erg} \mathrm{s}^{-1}$ at a distance of $12 \mathrm{kpc}$. Although the spectrum is similar to those of 
pulsars, there is no radio pulsar in the $3 \sigma$ circle responsible for the high luminosity. While the power-law electron spectrum with a cutoff for inverse Compton scattering would lead to a difficulty in the electron energy budget, the emission can be naturally explained by the hadronic interaction between the relativistic protons accelerated by the shock of SNR Kes 41 and the adjacent northwestern MC. By comparison with the hadronic interaction at the shock, which appears off the best-fit position of the source, illumination of the adjacent MC by the protons escaping from the shock front seems more consistent with observations. A list of Galactic thermal composite SNRs detected at $\mathrm{GeV} \gamma$-ray energies by Fermi-LAT is presented in this paper.

B.L. is grateful to Xia Fang, Ning-Xiao Zhang, and Zheng-Gao Xiong for the help about Fermi data analysis. We thank the support of NSFC grants 11233001 and 11403075 . This work has also benefited from 973 Program grant 2015CB857100, grant 20120091110048 from the Educational Ministry of China, and the grants from the 985 Project of NJU and the Advanced Discipline Construction Project of Jiangsu Province. This research has made use of the SIMBAD database, operated at CDS, Strasbourg, France.

\section{REFERENCES}

Abdo, A. A., et al. 2009, ApJ, 706, L1

—. 2010a, ApJ, 718, 348

—. 2010b, ApJ, 722, 1303

—. 2010c, Science, 327, 1103

—. 2010d, ApJ, 712, 459

—. 2010e, ApJS, 187, 460

—. 2013, ApJS, 208, 17

Acero, F., et al. 2015, ApJS, 218, 23

Ackermann, M., et al. 2013, Science, 339, 807

Aharonian, F. A., \& Atoyan, A. M. 1996, A\&A, 309, 917

Araya, M. 2013, MNRAS, 434, 2202 
-. 2014, MNRAS, 444, 860

Atwood, W. B., et al. 2009, ApJ, 697, 1071

Bell, A. R. 1978, MNRAS, 182, 147

Blandford, R. D., \& Cowie, L. L. 1982, ApJ, 260, 625

Brandt, T. J., \& Fermi-LAT Collaboration. 2013, Advances in Space Research, 51, 247

Brogan, C. L., \& Troland, T. H. 2001, ApJ, 550, 799

Byun, D.-Y., Koo, B.-C., Tatematsu, K., \& Sunada, K. 2006, ApJ, 637, 283

Castro, D., \& Slane, P. 2010, ApJ, 717, 372

Castro, D., Slane, P., Carlton, A., \& Figueroa-Feliciano, E. 2013, ApJ, 774, 36

Caswell, J. L. 2004, MNRAS, 349, 99

Chen, Y., Seward, F. D., Sun, M., \& Li, J.-t. 2008, ApJ, 676, 1040

Combi, J. A., Albacete-Colombo, J. F., \& Martí, J. 2008, A\&A, 488, L25

Combi, J. A., et al. 2010, A\&A, 523, A76

Ergin, T., Sezer, A., Saha, L., Majumdar, P., Chatterjee, A., Bayirli, A., \& Ercan, E. N. 2014, ApJ, 790, 65

Frail, D. A. 2011, Mem. Soc. Astron. Italiana, 82, 703

Gabici, S., Aharonian, F. A., \& Casanova, S. 2009, MNRAS, 396, 1629

Gaisser, T. K., Protheroe, R. J., \& Stanev, T. 1998, ApJ, 492, 219

Gelfand, J. D., Castro, D., Slane, P. O., Temim, T., Hughes, J. P., \& Rakowski, C. 2013, ApJ, 777, 148

Ginzburg, V. L., \& Syrovatskii, S. I. 1969, The origin of cosmic rays (New York: Gordon and Breach)

Hui, C. Y., Wu, E. M. H., Wu, J. H. K., Huang, R. H. H., Cheng, K. S., Tam, P. H. T., \& Kong, A. K. H. 2011, ApJ, 735, 115

Jiang, B., Chen, Y., Wang, J., Su, Y., Zhou, X., Safi-Harb, S., \& DeLaney, T. 2010, ApJ, 712, 1147 
Jones, T. W., et al. 1998, PASP, 110, 125

Koo, B.-C., Lee, J.-J., Seward, F. D., \& Moon, D.-S. 2005, ApJ, 633, 946

Koralesky, B., Frail, D. A., Goss, W. M., Claussen, M. J., \& Green, A. J. 1998, AJ, 116, 1323

Lande, J., et al. 2012, ApJ, 756, 5

Landecker, T. L., Pineault, S., Routledge, D., \& Vaneldik, J. F. 1989, MNRAS, 237, 277

Lemoine-Goumard, M., Grondin, M.-H., Acero, F., Ballet, J., Laffon, H., \& Reposeur, T. 2014, ApJ, 794, L16

Li, H., \& Chen, Y. 2010, MNRAS, 409, L35

-. 2012, MNRAS, 421, 935

McClure-Griffiths, N. M., Green, A. J., Dickey, J. M., Gaensler, B. M., Haynes, R. F., \& Wieringa, M. H. 2001, ApJ, 551, 394

Nolan, P. L., et al. 2012, ApJS, 199, 31

Ohira, Y., Murase, K., \& Yamazaki, R. 2011, MNRAS, 410, 1577

Pivato, G., et al. 2013, ApJ, 779, 179

Radhakrishnan, V., Goss, W. M., Murray, J. D., \& Brooks, J. W. 1972, ApJS, 24, 49

Reich, W., Fuerst, E., \& Arnal, E. M. 1992, A\&A, 256, 214

Reid, M. J. 1993, ARA\&A, 31, 345

Reynoso, E. M., \& Mangum, J. G. 2000, ApJ, 545, 874

Rho, J., \& Petre, R. 1998, ApJ, 503, L167

Rosado, M., Ambrocio-Cruz, P., Le Coarer, E., \& Marcelin, M. 1996, A\&A, 315, 243

Routledge, D., Dewdney, P. E., Landecker, T. L., \& Vaneldik, J. F. 1991, A\&A, 247, 529

Sedov, L. I. 1959, Similarity and Dimensional Methods in Mechanics (New York: Academic)

Seta, M., et al. 1998, ApJ, 505, 286

Tanaka, T., et al. 2011, ApJ, 740, L51 
Tang, X., \& Chevalier, R. A. 2014, ApJ, 784, L35

Uchida, K., Morris, M., \& Yusef-Zadeh, F. 1992, AJ, 104, 1533

Uchiyama, Y., Blandford, R. D., Funk, S., Tajima, H., \& Tanaka, T. 2010, ApJ, 723, L122

Wenger, M., et al. 2000, A\&AS, 143, 9

Whiteoak, J. B. Z., \& Green, A. J. 1996, A\&AS, 118, 329

Xing, Y., Wang, Z., Zhang, X., \& Chen, Y. 2014, ApJ, 781, 64

—. 2015, ApJ, 805, 19

Yuan, Q., Huang, X., Liu, S., \& Zhang, B. 2014, ApJ, 785, L22

Yuan, Q., Liu, S., \& Bi, X. 2012, The Astrophysical Journal, 761, 133

Zhang, G.-Y., Chen, Y., Su, Y., Zhou, X., Pannuti, T. G., \& Zhou, P. 2015, ApJ, 799, 103

Zou, W. Z., Hobbs, G., Wang, N., Manchester, R. N., Wu, X. J., \& Wang, H. X. 2005, MNRAS, 362, 1189 
Table 1. Fermi LAT Flux Measurements of Source A in the Kes 41 Region

\begin{tabular}{ccc}
\hline \hline $\begin{array}{c}E_{\mathrm{ph}}(\text { energy band }) \\
(\mathrm{GeV})\end{array}$ & $\begin{array}{c}E_{\mathrm{ph}}^{2} d N\left(E_{\mathrm{ph}}\right) / d E_{\mathrm{ph}}{ }^{\mathrm{a}} \\
\left(10^{-12} \mathrm{erg} \mathrm{cm}^{-2} \mathrm{~s}^{-1}\right)\end{array}$ & TS value \\
\hline $0.32(0.20-0.50)$ & $15.9 \pm 3.3 \pm 7.3$ & 39 \\
$0.71(0.50-1.00)$ & $24.4 \pm 4.6 \pm 9.9$ & 113 \\
$1.73(1.00-3.00)$ & $21.3 \pm 1.8 \pm 4.6$ & 224 \\
$5.48(3.00-10.0)$ & $10.7 \pm 1.2 \pm 1.7$ & 105 \\
$17.3(10.0-30.0)$ & $2.4 \pm 1.0 \pm 0.3$ & 9 \\
$94.9(30.0-300)$ & $\leq 2.9^{\mathrm{b}}$ & 2 \\
\hline
\end{tabular}

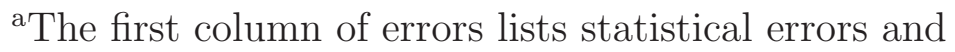
the second lists systematic errors.

b The $95 \%$ upper limit.

Table 2. Model Parameters for the Emissions of SNR Accelerated Particles

\begin{tabular}{|c|c|c|c|c|c|c|}
\hline & & $\alpha_{\mathrm{e}}$ & & $\begin{array}{c}E_{\text {e,cut }} \\
(\mathrm{GeV})\end{array}$ & $\begin{array}{c}W_{\mathrm{e}}(>1 \mathrm{GeV}) \\
\left(10^{51} \mathrm{erg}\right)\end{array}$ & \\
\hline \multirow[t]{2}{*}{ case A } & & 2.0 & & 400 & 1.3 & \\
\hline & & $\alpha_{\mathrm{p}}$ & 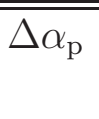 & $\begin{array}{c}E_{\mathrm{b}} \\
(\mathrm{GeV})\end{array}$ & $\begin{array}{c}n_{\mathrm{t}} E_{51}^{-1} W_{\mathrm{p}}(>1 \mathrm{GeV}) \\
\left(10^{51} \mathrm{erg} \mathrm{cm}^{-3}\right)\end{array}$ & \\
\hline \multirow[t]{2}{*}{ case B } & & 2.0 & 1.2 & 18 & 7 & \\
\hline & $\begin{array}{c}t_{\text {age }} \\
(\mathrm{kyr})\end{array}$ & $\overline{\alpha \alpha_{\mathrm{p}}}$ & $\bar{\delta}$ & $\bar{\chi}$ & $\begin{array}{l}\Delta R_{c} \\
(\mathrm{pc})\end{array}$ & $\begin{array}{c}M_{\mathrm{cl}} \\
\left(10^{4} M_{\odot}\right) \\
\end{array}$ \\
\hline \multirow[t]{4}{*}{ case $\mathrm{C}$} & 4 & 2.4 & 0.7 & 0.07 & 5 & 4.5 \\
\hline & 10 & 2.4 & 0.7 & 0.03 & 10 & 11 \\
\hline & 100 & 2.4 & 0.7 & 0.004 & 13 & 18 \\
\hline & $\begin{array}{c}t_{\text {age }} \\
(\mathrm{kyr})\end{array}$ & $\overline{\alpha_{\mathrm{p}}}$ & 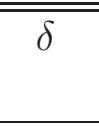 & $\chi$ & $\begin{array}{l}R_{c} \\
(\mathrm{pc})\end{array}$ & $\begin{array}{c}M_{\mathrm{cl}} \\
\left(10^{4} M_{\odot}\right) \\
\end{array}$ \\
\hline \multirow[t]{3}{*}{ case D } & 10 & 2.4 & 0.7 & 0.25 & 15 & 18 \\
\hline & 10 & 2.4 & 0.7 & 0.45 & 20 & 40 \\
\hline & 100 & 2.4 & 0.7 & 0.05 & 20 & 40 \\
\hline
\end{tabular}


Table 3. Parameters of the $\gamma$-ray Emission of the Galactic Thermal Composite SNRs Obtained from Fermi-LAT Observation

\begin{tabular}{|c|c|c|c|c|c|}
\hline Source & $\begin{array}{l}\text { Distance } \\
(\mathrm{kpc})\end{array}$ & $\Gamma$ & $\begin{array}{c}L_{1-100 \mathrm{GeV}} \\
\left(10^{35} \mathrm{erg} \mathrm{s}^{-1}\right)\end{array}$ & MC interaction ${ }^{\mathrm{a}}$ & References \\
\hline G6.4-0.1(W28) & 2.0 & $2.74 \pm 0.06^{\mathrm{b}}$ & 1.0 & $\mathrm{Y}$ & 1,2 \\
\hline $\mathrm{G} 31.9+0.0(3 \mathrm{C} 391)$ & 7.2 & $2.50 \pm 0.04^{\mathrm{b}}$ & 4.0 & $\mathrm{Y}$ & 3,4 \\
\hline G34.7-0.4(W44) & 2.8 & $3.02 \pm 0.10^{\mathrm{b}}$ & 2.7 & $\mathrm{Y}$ & 5,6 \\
\hline G43.3-0.2(W49B) & 8 & $2.29 \pm 0.02^{\mathrm{c}}$ & 8.0 & $\mathrm{Y}$ & 7,8 \\
\hline G49.2-0.7(W51C) & 6 & $2.5 \pm 0.1^{\mathrm{b}}$ & 4.4 & $\mathrm{Y}$ & 9,10 \\
\hline G89.0+4.7(HB 21) & 1.7 & $2.33 \pm 0.03^{\mathrm{c}}$ & 0.13 & $\mathrm{Y}$ & 11,12 \\
\hline G189.1+3.0(IC 443) & 1.5 & $2.61 \pm 0.04^{\mathrm{b}}$ & 1.0 & $\mathrm{Y}$ & 5,13 \\
\hline G304.6+0.1(Kes 17) & 9.7 & $2.0 \pm 0.3^{\mathrm{c}}$ & 12 & $\mathrm{Y}$ & 14,15 \\
\hline G327.4+0.4(Kes 27$)$ & 4.3 & $2.5 \pm 0.1^{\mathrm{c}}$ & 0.24 & & 16,17 \\
\hline G337.8-0.1(Kes 41) & 12 & $2.38 \pm 0.03^{\mathrm{c}}$ & 7.7 & $\mathrm{Y}$ & 18 \\
\hline G348.5+0.1(CTB 37A) & 11.3 & $2.19 \pm 0.07^{\mathrm{c}}$ & 7.8 & $\mathrm{Y}$ & $19,20,21$ \\
\hline G357.7-0.1(MSH 17-39) & 12 & $2.5 \pm 0.3^{\mathrm{c}}$ & 5.8 & $\mathrm{Y}$ & 22,23 \\
\hline G359.1-0.5 & 7.6 & $2.60 \pm 0.05^{\mathrm{c}}$ & 4.0 & $\mathrm{Y}$ & 24,25 \\
\hline G0.0+0.0(Sgr A East) $(?)^{\mathrm{d}}$ & 8.0 & $2.32 \pm 0.03^{\mathrm{c}}$ & 8.7 & $\mathrm{Y}$ & 26,23 \\
\hline G132.7+1.3(HB 3) $(?)^{\mathrm{d}}$ & 2.2 & $2.30 \pm 0.11^{\mathrm{c}}$ & 0.04 & $Y ?$ & 27,23 \\
\hline $\mathrm{G} 156.2+5.7(?)^{\mathrm{d}}$ & 3 & $2.35 \pm 0.09^{\mathrm{c}}$ & 0.26 & & 28,23 \\
\hline G290.1-0.8(MSH 11-61A) $(?)^{\mathrm{d}}$ & 7 & $\sim 2.28^{\mathrm{c}}$ & 1.5 & $?$ & 22,23 \\
\hline G160.9+2.6(HB 9) $(?)^{\mathrm{e}}$ & 1.0 & $2.30 \pm 0.05^{\mathrm{c}}$ & 0.013 & $?$ & 29 \\
\hline $\mathrm{G} 166.0+4.3(?)^{\mathrm{e}}$ & 4.5 & $2.27 \pm 0.1^{\mathrm{c}}$ & 0.11 & $?$ & 30,31 \\
\hline
\end{tabular}

Note. - (1) Frail 2011; (2) Abdo et al. 2010a; (3) Radhakrishnan et al. 1972; (4) Ergin et al. 2014; (5) Seta et al. 1998; (6) Abdo et al. 2010c; (7) Brogan \& Troland 2001; (8) Abdo et al. 2010b; (9) Koo et al. 2005 (10) Abdo et al. 2009; (11) Byun et al. 2006; (12) Pivato et al. 2013 (13) Abdo et al. 2010d; (14) Combi et al. 2010; (15) Gelfand et al. 2013; (16) McClure-Griffiths et al. 2001; (17)Xing et al. 2015; (18) Zhang et al. 2015; (19) Reynoso \& Mangum 2000; (20) Castro \& Slane 2010; (21) Brandt \& Fermi-LAT Collaboration 2013; (22) Rosado et al. 1996; (23) Acero et al. 2015; (24)Uchida et al. 1992; (25) Hui et al. 2011; (26) Reid 1993; (27) Routledge et al. 1991; (28) Reich et al. 1992; (29) Arava 2014; (30)Landecker et al. 1989; (31) Arava 2013;

a Adopted from Jiang et al. 2010 SNR-MC association table.

${ }^{\mathrm{b}}$ The photon index above the break energy for broken power-law spectrum.

${ }^{\mathrm{c}}$ The photon index of single power-law spectrum.

${ }^{\mathrm{d}}$ Question mark: the association of the detected $\gamma$-ray emission with the SNR is uncertain.

'Question mark: not listed in the latest 3FGL catalogue Acero et al. 2015). 


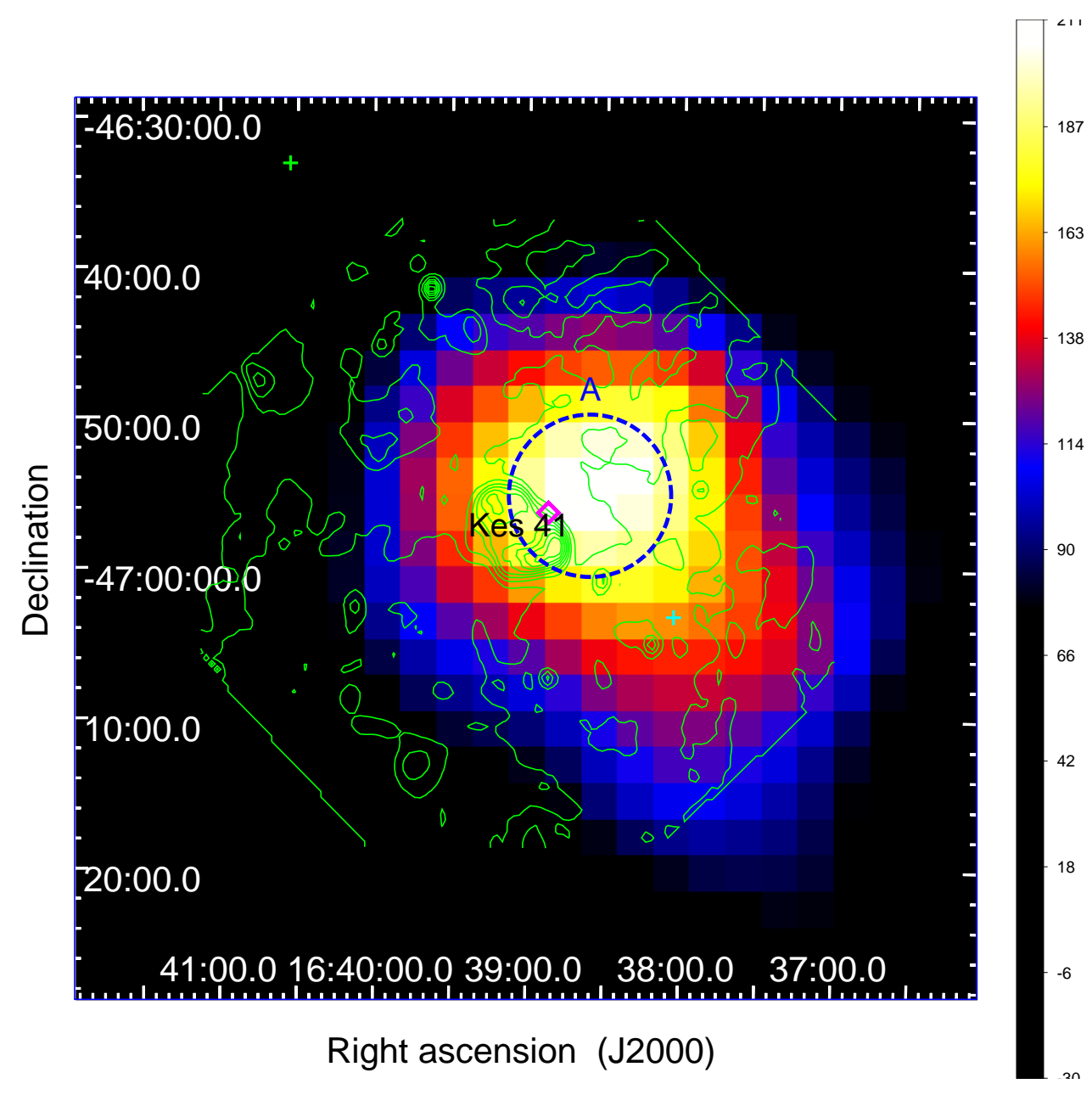

Fig. 1. - TS map $(2-300 \mathrm{GeV})$ of $1^{\circ} \times 1^{\circ}$ region centered at Kes 41 . All sources except 2FGL J1638.0-4703c have been subtracted. The green cross labels the position of a 2FGL source, the cyan cross labels the position of 2FGL J1638.0-4703c, and the dashed blue circle indicates the $3 \sigma$ error range of the best-fit position for the residual emission found in the Kes 41 region. The image is overlaid with the MOST $843 \mathrm{MHz}$ radio contours (in green) (at seven linear scale levels between 0.00 and $0.79 \mathrm{Jy}_{\text {beam }}{ }^{-1}$; from Whiteoak \& Green (1996)). The magenta diamond represents the $\mathrm{OH}(1720 \mathrm{MHz})$ maser spot (Koralesky et al. 1998). 


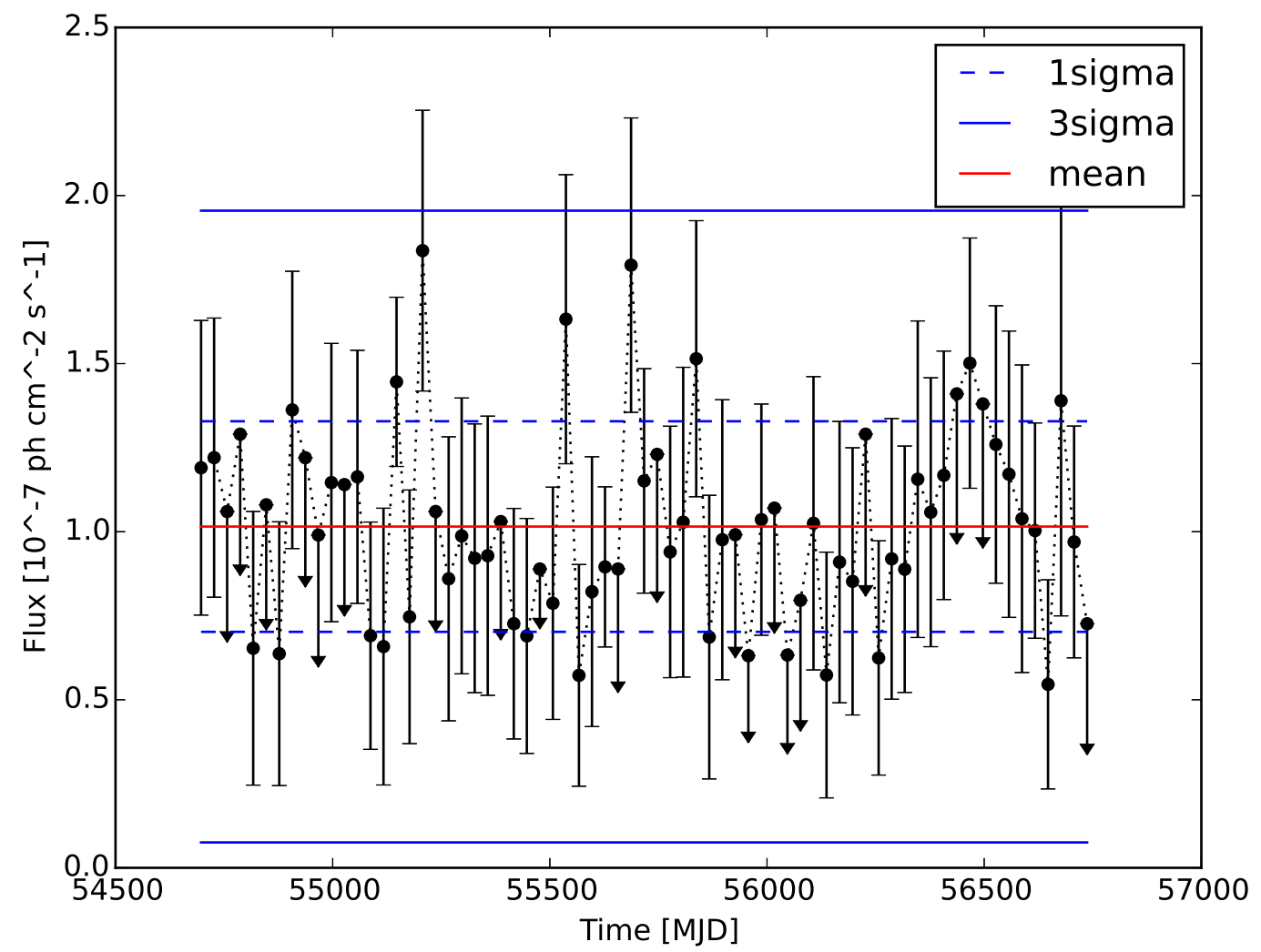

Fig. 2.- Monthly $\gamma$-ray light curve of source A in the energy range of $0.2-300 \mathrm{GeV}$. 


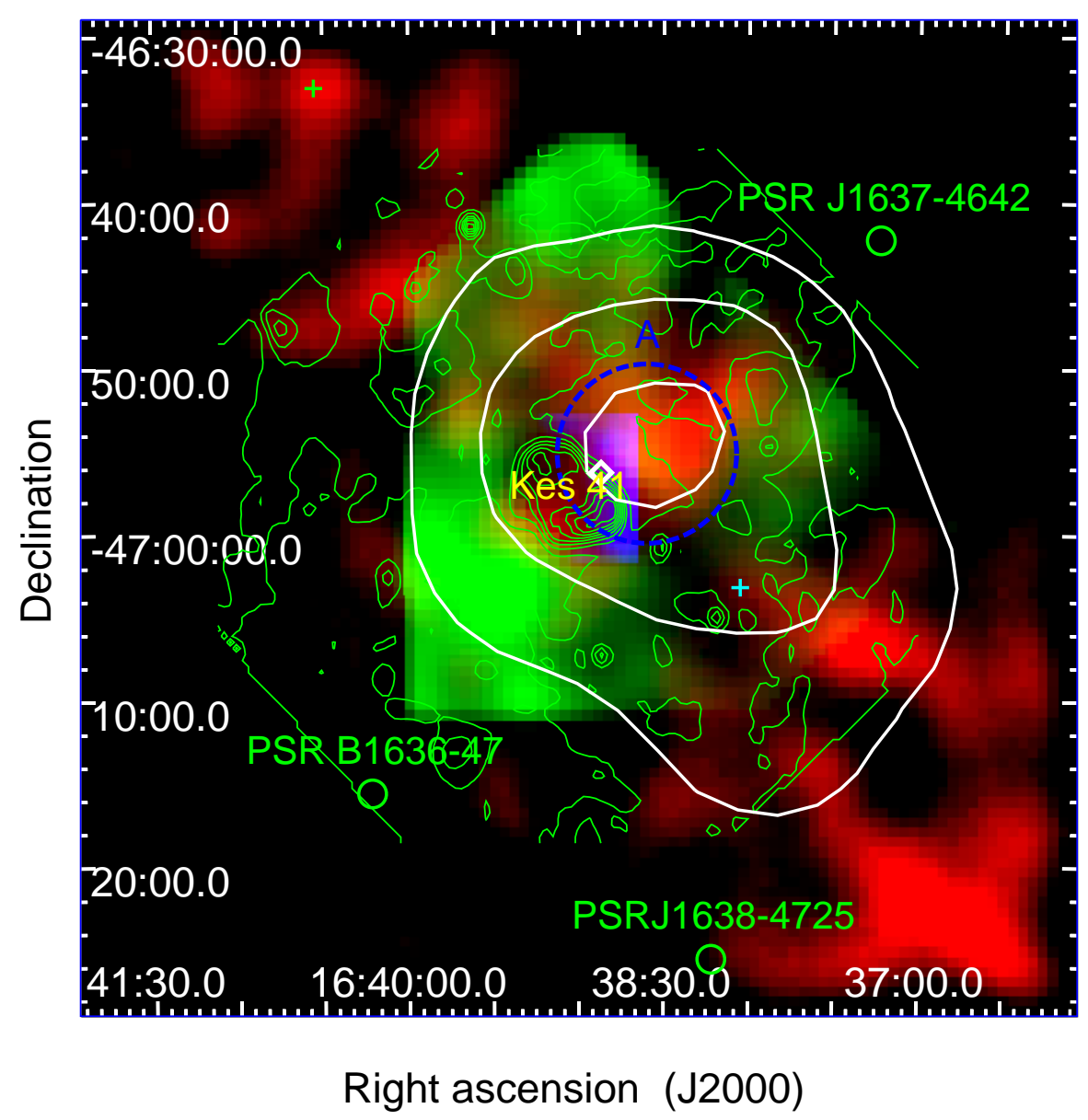

Fig. 3.- Tri-color image of Kes 41 in multiwavelengths. Red: Fermi-LAT 2-300GeV counts map centered at SNR Kes 41, smoothed with a Gaussian of width 0.6 (per pixel bin representing 0.01). Blue: ${ }^{12} \mathrm{CO}(J=1-0)$ integrated emission $\left(V_{\mathrm{LSR}}=-70\right.$ to $\left.-40 \mathrm{~km} \mathrm{~s}^{-1}\right)$ with a field of view of $11^{\prime} \times 10^{\prime}$. Green: H I line emission from SGPS integrated map $\left(V_{\mathrm{LSR}}=-55\right.$ to $\left.-50 \mathrm{~km} \mathrm{~s}^{-1}\right)$. The green contours and the green and cyan crosses are the same as in Fig,1. The white curves show the TS $=100,144$ and 196 contours (which correspond to significance $10 \sigma, 12 \sigma$ and $14 \sigma$, respectively). The white diamond indicates the location of the $1720 \mathrm{MHz} \mathrm{OH}$ maser (Koralesky et al. 1998) and the green circles label the positions of known pulsars. The dashed blue circle indicates the $3 \sigma$ error circle of the best-fit position of source A. 


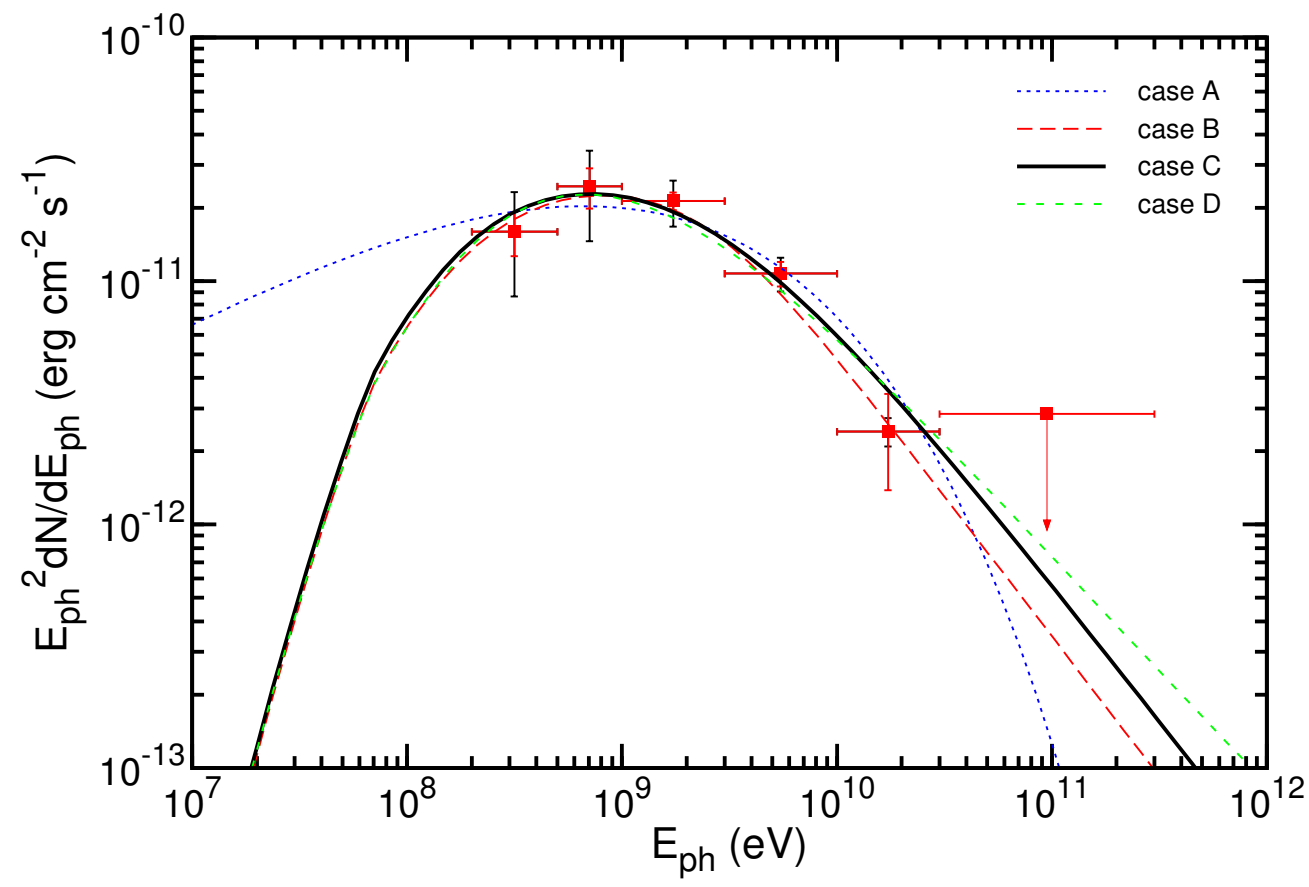

Fig. 4.- Fermi $\gamma$-ray spectral energy distribution of source A fit with various models (see text). Systematic errors (see Section 3.4) are indicated by black bars and the statistical errors are indicated by red bars. 